\title{
Cardiomyocyte Derived miR-328 Promotes Cardiac Fibrosis by Paracrinely Regulating Adjacent Fibroblasts
}

\author{
Dandan Zhao ${ }^{\mathrm{a}}$ Cui Lib He Yan ${ }^{\mathrm{a}}$ Tianyu Lia Ming Qian ${ }^{\mathrm{a}} \quad$ Nan Zheng ${ }^{\mathrm{a}}$ \\ Hua Jianga Li Liua Bozhi Xua Qiuxia Wu ${ }^{a}$ Xuelian Lia Haihai Lianga \\ Hongli Shan ${ }^{a}$
}

aDepartment of Pharmacology (State-Province Key Laboratories of Biomedicine-Pharmaceutics of China, Key Laboratory of Cardiovascular Research, Ministry of Education), College of Pharmacy, Harbin Medical University, Harbin, Heilongjiang, 'Department of Physiology, Xuzhou Medical University, Xuzhou, Jiangsu, P. R. China

\section{Key Words \\ miR-328 • Fibrosis • Transfer • Paracrine}

\begin{abstract}
Background/Aims: In our previous study, we demonstrated that elevated expression of miR328 is a potent determinant of cardiac fibrosis during myocardial infarction (MI). In the present study, histological examination revealed progressive fibrosis in transgenic mice overexpressing cardiomyocyte-specific miR-328. This study investigated whether the transfer of miR-328 from cardiomyocytes (CMs) to cardiac fibroblasts (CFs) in a paracrine manner contributes to myocardial fibrosis. Methods: Myocardial infarction was established by the occlusion of the left coronary artery. Masson's trichrome staining and collagen assays were used to evaluate the progression of fibrosis. The vesicles and translocation of miR-328 in a co-culture assay system were respectively observed using transmission electron microscopy (TEM) and immunofluorescence staining (IF). Real-time PCR was employed to detect the level of miR-328, Col $1 \alpha 1$ and Col $3 \alpha 1$. The protein expression of Col1 $\alpha 1$, TGF- $\beta$ RIII, p-smad2/3 (phosphorylatedsmad2/3) and TGF- $\beta 1$ were probed using western blot analysis. Results: Cardiomyocytespecific miR-328 overexpressing transgenic (TG) mice showed enhanced collagen deposition and provoked cardiac fibrosis by the activation of the TGF- $\beta 1$ pathway, and this effect was abrogated after knockdown of endogenous miR-328 in mice. Correspondingly, the expression of miR-328 was increased in CFs co-cultured with CMs transfected with miR-328 mimics, likely in a paracrine manner. The cardiomyocyte-mediated augmentation of miR-328 contributes to fibrogenesis in CFs, and this pro-fibrotic effect was reversed after the transfection of miR-328 inhibitor in CFs. Conclusion: A novel molecular mechanism for miR-328 derived from CMs as a paracrine signaling mediator of cardiac fibrogenesis further demonstrates that miR-328 is a potential therapeutic target.

D. Zhao and C. Li contributed equally to this work.
\end{abstract}

Hongli Shan

and Haihai Liang 


\section{Introduction}

Cardiac fibrosis is characterized by the abnormal proliferation of cardiac fibroblasts and interstitial deposition of extracellular matrix (ECM) in the myocardium [1]. As a typical pathologic indication, cardiac fibrosis is accompanied by a wide range of cardiovascular disorders, such as myocardial infarction, cardiac hypertrophy and atrial fibrillation (AF).

Cardiac fibroblasts and cardiomyocytes have crucial synergistic effects [2] in maintaining homeostasis and responding to injuries [3]. The communication of CMs and CFs depends on interactions, including the paracrine pathway, extracellular matrix interactions, electrical modulation and structural junctions [4]. An increasing number of studies have revealed that the cross talk between CFs and CMs is conducive to pathological processes via effects on extracellular matrix composition and interstitial fibrosis [5].

miRNAs are well-known pivotal regulators participating in the initiation and progression of cardiovascular disease, including arrhythmia [6], cardiac hypertrophy [7], heart failure, and cardiac fibrosis [8]. Recent studies indicate that miRNAs could be secreted from a donor cell into the extracellular medium and subsequently permeate a recipient cell [9]. miRNAs move from smooth muscle cells to endothelial cells [10], and cancer cells to immune cells [11] via microvesicles or exosomes to mediate target gene repression resulting in physiological changes and pathological phenotypes [12,13]. Notably, whether the miRNAs could be transferred from cardiomyocytes to fibroblasts, facilitating fibrogenesis has rarely been reported.

In a previous study, we generated a transgenic (TG) mouse line with the cardiomyocytespecific overexpression of miR-328 and revealed that these TG mice exhibit atrial fibrillation and cardiac hypertrophy $[14,15]$. Notably, histological examination suggested progressive fibrosis in the hearts of miR-328 TG mice in the present study. This study was designed to determine whether miR-328 derived from CMs were transferred to CFs to promote cardiac fibrogenesis.

\section{Materials and Methods}

\section{Generation of miR-328 TG mice}

The miR-328 transgenic mice were generated as previously described [15]. A fragment containing the pre-miR-328 sequence was subcloned into the Bluescript vector (Promega Co. Ltd., Madison, Wisconsin, USA) carrying the cardiac-specific $\alpha$-myosin heavy chain ( $\alpha \mathrm{MHC}$ ) promoter. Transgenic mice carrying a mismatched pre-miR-328 sequence were generated as a negative control. Each egg was individually microinjected with the DNA fragment at a concentration of $3 \mathrm{ng} / \mu \mathrm{L}$ and subsequently implanted into bilateral oviduct of pseudo-pregnant female mice. Genomic DNA from tail tissues of the transgenic mice was extracted to verify the presence of the miR-328 transgene. The cholesterol-conjugated miR-328 antisense (antagomiR-328) and negative control (antagomiR-NC) were purchased from RiboBio Co., Ltd. (Guangzhou, China). AntagomiR-328/antagomiR-NC was administered through tail vein injection at a dose of $40 \mathrm{mg} / \mathrm{kg}$ once a day for three consecutive days.

\section{Generation of miR-328 knockdown (KD) mice}

The same procedures were followed as described above for miR-328 [15]. A fragment containing 6 antimiR-328 antisense units (Shanghai Biological Engineering Inc., Shanghai, China) was synthesized and subsequently subcloned into the Bluescript vector (Promega Co. Ltd., Madison, Wisconsin, USA) carrying the cardiac-specific MHC promoter. Endogenous miR-328 was verified as knocked down.

\section{Mouse model of myocardial infarction (MI)}

Adult male (22 - 25 g) C57BL/6 wild-type (WT) mice and KD (knockdown of miR-328) mice used in the present study were maintained under controlled conditions. The mice were randomly divided into sham and MI groups. MI surgery was performed as previously described [16]. The mice were anaesthetized with pentobarbital $(40 \mathrm{mg} / \mathrm{kg})$ and subjected to an open chest procedure. The left anterior descending 


\section{Cellular Physiology Cell Physiol Biochem 2018;46:1555-1565 and Biochemistry Publisnec onI0048: Apro1 Z4, $2018 \quad \begin{aligned} & \text { DOI 1018 2018 The Author(s). Published by S. Karger AG, Basel } \\ & \text { www.karger.com/cpb }\end{aligned}$ \\ Zhao et al.: Cardiomyocytes-Derived MiR-328 Conduce to Cardiac Fibrosis}

coronary artery was consistently occluded with a 6-0 ligature. An ECG recorder (BL-420, Chengdu TME Technology Co., Ltd., China) continuously recorded standard limb lead II ECG to diagnose the MI model. Sham animals for control underwent the same open chest procedures without coronary artery occlusion. All experimental procedures were performed in accordance with the Institutional Animal Care and Use Committee of Harbin Medical University, P.R. China. The protocol was approved through the Experimental Animal Ethic Committee of Harbin Medical University. One week after occlusion, the mice were sacrificed, and the hearts were removed. The $1-2 \mathrm{~mm}$ areas between the infarct and normal tissues were selected as peri-infarct regions for the following experiments.

\section{Antisense oligo-miR-328 injection}

AntagomiR-328 (antagomiR) and mutant-antagomiR-328 (M-antagomiR) at a dose of $40 \mathrm{mg} / \mathrm{kg}$ were delivered into TG mice through tail vein injection for 3 consecutive days as previously described [15]. One week after injection, the mice were sacrificed, and the hearts were removed. The $1-2 \mathrm{~mm}$ areas between the infarct and normal tissues were selected as peri-infarct regions for the following experiments.

\section{Echocardiography and Infarct size}

7 days after the MI, the mice were weighed and anesthetized as previously mentioned. The left ventricular function of mice were examined with Vivo 2100 rodent echocardiographic imaging system (Visualsonics, Toronto, Canada) equipped with a $30 \mathrm{MHz}$ microscan transducer. Fractional shortening (FS) and ejection fraction (EF) were evaluated. The ventricular tissues were obtained and stained with $1 \% 2$, 3,5-Triphenyltetrazolium chloride (TTC) solution at $37^{\circ} \mathrm{C}$ for $20 \mathrm{~min}$. Images obtained at low magnification. Image analysis software Image Pro Plus (version 6.0, Media Cybernetics) was used for caculating the ratio of ventricular infarction area to ventricular total area.

\section{Masson's trichrome staining}

The 1 - $2 \mathrm{~mm}$ areas between the infarct region and normal tissue were selected as peri-infarct regions. The tissues were immediately dissected and immersed in $4 \%$ paraformaldehyde for $24 \mathrm{~h}$ and subsequently stained with Masson's Trichrome. The collagen fibres were stained in blue, the nuclei were stained in black, and the cytoplasm was stained in red. Three areas were analysed in each slide, and each area was divided into 100 squares. The number of collagen points (blue stain) was scored as 1 (present) or 0 (absent). The results are shown as the percentage of area occupied by fibrosis to the total area. Fibrotic tissue was quantified using image analysis software (Image Pro Plus 6.0, Meida Cybernetics LP) as previously described [17].

\section{Measurement of collagen content}

Total collagen content was measured using the Sircol Collagen Assay Kit (Biocolour.co., County Antrim, UK) according to the manufacturer's instructions. Each sample was incubated with $200 \mu \mathrm{L}$ of cold separation reagent for $24 \mathrm{~h}$ at $4^{\circ} \mathrm{C}$. After centrifugation at $12000 \mathrm{rpm}$ for $10 \mathrm{~min}, 100 \mu \mathrm{L}$ of each supernatant was used in subsequent assays. One millilitre of Sircol dye reagent was added to each sample, and the solution was gently shaken at $4^{\circ} \mathrm{C}$ for $30 \mathrm{~min}$. The supernatant was discarded after centrifugation, and the pellet was resuspended in $250 \mu \mathrm{L}$ of the alkali reagent included in the kit. A $200 \mu \mathrm{L}$ aliquot of solution was collected and assessed at $540 \mathrm{~nm}$ using a spectrophotometer (SUNRISE, Switzerland). A standard curve was constructed using collagen standards [18]. The amount of protein was assessed at $562 \mathrm{~nm}$ using a BCA protein assay kit (Thermo Fisher Scientific Inc., Waltham, MA., USA).

\section{Transmission electron microscopy (TEM)}

The CMs were fixed with $2.5 \%$ glutaraldehyde $(\mathrm{pH} 7.4)$ at $4^{\circ} \mathrm{C}$ overnight, and subsequently fixed in $1.0 \%$ osmium tetroxide for $1 \mathrm{~h}$. The monolayer was blocked in uranyl acetate as previously described [19], and observed under an electron microscope (JEM-1220, JEOL, Ltd., Tokyo, Japan).

\section{Cell culture and treatment}

Primary neonatal rat cardiomyocytes (CMs) and cardiac fibroblasts (CFs) were isolated from 1- to 3-day-old Wistar rats as previously described [20]. Transwell chambers (Millipore Corporation, Massachusetts, USA) were used for the indirect co-culture assay. The CFs were seeded onto $25-\mathrm{cm}^{2}$ cell culture flasks containing $2 \mathrm{~mL}$ DMEM (Biological Industries, Israel) supplemented with 10\% fetal bovine 


\section{Cellular Physiology Cell Physiol Biochem 2018;46:1555-1565 and Biochemistry Published online: April 24, $2018 \quad \begin{aligned} & \text { DOI 1015/2018 The Author(s). Published by S. Karger AG, Basel } \\ & \text { www.karger.com/cpb }\end{aligned}$ \\ Zhao et al.: Cardiomyocytes-Derived MiR-328 Conduce to Cardiac Fibrosis}

serum (FBS) and $1 \%$ penicillin/streptomycin at $37^{\circ} \mathrm{C}$ in $5 \% \mathrm{CO}_{2}$. The CMs were seeded onto the transwell chambers with $1.5 \mathrm{~mL}$ of DMEM supplemented with $10 \% \mathrm{FBS}$ and $1 \%$ penicillin/streptomycin in the upper layer and with $2.5 \mathrm{~mL}$ of DMEM supplemented with $10 \% \mathrm{FBS}$ and 1\% penicillin/streptomycin in the lower layer of the cell culture flasks at $37^{\circ} \mathrm{C}$ in $5 \% \mathrm{CO}_{2}$.

After $48 \mathrm{~h}$ of culturing, the miRNA (GenePharma Integrated DNA Technologies, Inc., Suzhou, Jiangsu, China) and X-treme GENE siRNA transfection reagent (Roche Applied Science, Mannheim, Germany) were separately mixed with $500 \mu \mathrm{L}$ of Opti-MEM (Thermo Fisher Scientific Inc., Waltham, MA., USA) for $10 \mathrm{~min}$ on ice. The two mixtures were subsequently combined and incubated for $20 \mathrm{~min}$ on ice. The CMs were respectively transfected with the miR-328 mimic (miR-328, $100 \mathrm{nmol} / \mathrm{L}$ ) or mimic negative control (miR-NC, $100 \mathrm{nmol} / \mathrm{L}$ ), and the CFs were separately transfected with antisense oligonucleotide-miR-328 inhibitor (AMO-328, $100 \mathrm{nmol} / \mathrm{L}$ ) or antisense oligonucleotide-miR-negative control inhibitor (AMO-NC, $100 \mathrm{nmol} / \mathrm{L}$ ) according to the manufacturer's instructions, as previously described [8].

Cy3-labelled miR-328 transfection was performed using the same procedure described above. The CMs were transfected with the Cy3-labelled miR-328 (100 nmol/L). After $12 \mathrm{~h}$ transfection, the medium was removed, and the chambers were washed $2-3$ times with PBS. Simultaneously, the transwells embedded with CMs were moved to culture flasks containing CFs co-cultured for $48 \mathrm{~h}$ with DMEM supplemented with $10 \% \mathrm{FBS}$ and $1 \%$ penicillin/streptomycin at $37^{\circ} \mathrm{C}$ in $5 \% \mathrm{CO}_{2}$.

Z-vad-FMK (Z-vad; $10 \mathrm{mmol} / \mathrm{L}$; Selleck Chemicals, Houston, Texas, US) was added when CMs and CFs co-cultured until the cells were extracted for subsequent experiments.

\section{Cell Counting Kit-8 (CCK-8) assay}

Cell viability was detected with Cell Counting Kit-8 (CCK-8; Dojindo Laboratories, Tokyo, Japan). Cardiomyocytes were cultured and treated in 96-well microplates. Cells were further incubated and measured with CCK- 8 at a concentration of $10 \%$ for 4 hours, and absorbance values were collected at 450 $\mathrm{nm}$. Cell viability rate was calculated as per the following equation: viability rate $=[(\mathrm{As}-\mathrm{Ab}) /(\mathrm{Ac}-\mathrm{Ab})]$, where As is the treated cell absorbance, Ac is the untreated cell absorbance and $\mathrm{Ab}$ is the background absorbance nothing but DMEM added. All experiments were repeated at least 3 times independently.

\section{Real-time PCR analysis}

Total RNA was extracted from heart tissues, co-cultured cells and medium using TRIzol reagent (Thermo Fisher Scientific Inc., Waltham, MA., USA) according to the manufacturer's instructions [17]. The mirVanamiRNA isolation kit (Applied Biosystems, foster city CA, USA) was used for quantification of miR-328 according to the manufacturer's instructions as previously described [15]. Real-time PCR was performed using the ABI 7500 Fast Real-Time PCR System (Applied Biosystems, Foster City CA, USA). GAPDH and U6 were respectively used as internal controls for mRNA and miRNA quantification.

\section{Western blot analysis}

Total protein samples were extracted from the tissues and co-cultured CFs as previously described [21]. Briefly, tissues and cells were lysed with lysis buffer (Beyotime Institute of Biotechnology, Shanghai, China). The concentration was measured using a bicinchoninic acid kit (Beyotime Institute of Biotechnology, Shanghai, China) according to the manufacturer's instructions. Equal amounts of $50 \mu \mathrm{g}$ of protein were fractionated on 10\% SDS-PAGE gels and transferred to nitrocellulose membranes (Millipore Corporation, Massachusetts, USA). The primary antibodies against TGF- $\beta 1$, TGF- $\beta$ RIII and Col $1 \alpha 1$ were rabbit polyclonal antibodies purchased from Cell Signaling Technology (Cell Signaling Technology, Inc., USA). With antiGAPDH antibody (Kangchen. Co. Inc., Beijing, China) serving as an internal control, the immunoreactivity was detected using the Odyssey v1.2 Infrared Imaging System (Gene Company Ltd., Hong Kong, China). The bands were quantified by measuring the intensity for each group.

\section{Co-immunoprecipitation (CO-IP)}

Tissues were lysed in RIPA buffer (Beyotime Institute of Biotechnology, Shanghai, China) and subsequently centrifuged at $12000 \times$ g for $15 \mathrm{~min}$ as previously described [22]. The supernatant protein was collected, and the primary antibody and IgG were mixed and incubated with protein A/G agarose beads at $4^{\circ} \mathrm{C}$ on a shaker overnight. The mixed liquor was centrifuged at $3000 \times \mathrm{g}$ for $5 \mathrm{~min}$, and the pellet was washed 3 times with PBS. The appropriate amount of loading buffer was added to the pellet, followed by boiling for 


\section{Cellular Physiology Cell Physiol Biochem 2018;46:1555-1565

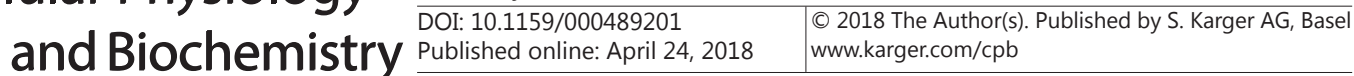 \\ Zhao et al.: Cardiomyocytes-Derived MiR-328 Conduce to Cardiac Fibrosis}

$7 \mathrm{~min}$ and centrifugation to dissociate the protein from the pellets. The supernatants were subsequently analysed using western blotting.

Immunofluorescence (IF)

The co-cultured CFs were immunohistochemically examined as previously described [19]. Briefly, the CFs were fixed in cool methanol for $10 \mathrm{~min}$ and penetrated with $0.4 \%$ Triton X-100 (Sigma-Aldrich Inc., St. Louis, MO, USA) and 1\% BSA (Sigma-Aldrich Inc., St. Louis, MO, USA) diluted with PBS for $1 \mathrm{~h}$ at room temperature. The cells were blocked with goat serum for $1 \mathrm{~h}$ at room temperature and subsequently incubated with primary antibodies against $\alpha$-SMA (Cell Signaling Technology, Inc., Boston, USA) and p-smad2/3 (Cell Signaling Technology, Inc., Boston, USA).

The fluorescent stain 4', 6-diamidino-2-phenylindole (DAPI, Thermo Fisher Scientific Inc., Waltham, MA., USA) was used to counterstain the nucleus for $5 \mathrm{~min}$ at room temperature. Immunofluorescence was visualized using a fluorescence microscope (Nikon 80i, Tokyo, Japan).

\section{Statistical analyses}

The data are presented as the means \pm SEM. Nonparametric test (the Wilcoxon test for two groups and Kruskal-Wallis test for multiple groups) was applied when normality could not be determined as a result of small size sample. The Student's t-test was used for comparisons between two groups once normality had been determined. ANOVA (Bonferroni's Multiple Comparison Test) was applied to statistical comparisons among multiple groups. A two-tailed $P<0.05$ was considered significantly different. All graphs were drawn using GraphPad Prism 5.0.

\section{Results}

Cardiomyocyte-specific overexpression of miR-328 caused cardiac fibrosis in mice

Based on our previous studies [15], the fibrosis in cardiomyocyte-specific overexpression of miR-328 TG mice was examined as the first step. As shown in Fig. 1A, compared with the miR-328 expression in WT mice, miR-328 expression was increased in TG mice according to real-time PCR analyses. This increased expression was accompanied by massive collagen deposition and fibrosis (Fig. 1B and 1C). Additionally, miR-328 inhibition by the administration of antagomiR-328 alleviated collagen deposition and fibrosis in TG mice, whereas antagomiR-NC did not (Fig. 1B and 1C).

To further evaluate whether miR-328 inhibition induces resistance to fibrogenesis, we generated a miR-328 antisense TG mouse model carrying the cardiac-specific $\alpha$-MHC pro-

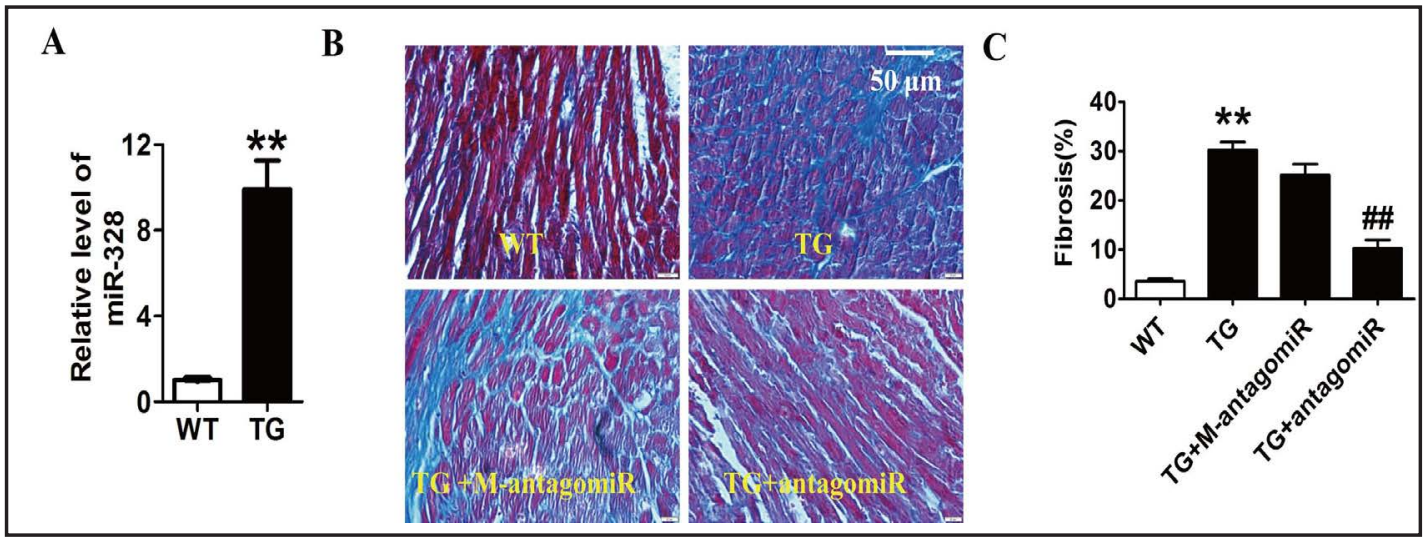

Fig. 1. Cardiomyocyte-specific overexpression of miR-328 caused cardiac fibrosis, and knockdown of miR328 alleviates cardiac fibrosis in mice. (A) Increased expression of miR-328 in TG mice compared with WT mice. $n=7$. (B) Representative sections showing that cardiac fibrosis in miR-328 TG mice was increased, whereas the fibrotic phenotype was attenuated after silencing miR-328 using antagomiR-328. (C) Crosssectional areas of fibrous tissue were analysed. $\mathrm{n}=5$. ${ }^{* *} \mathrm{p}<0.01 \mathrm{vs}$. WT; \#\#p<0.01 vs. TG. 
moter to genetically knock down endogenous miR328, designated KD mice. As shown in Fig. 2A, miR328 was decreased in KD mice according to real-time PCR analyses. Moreover, after myocardial infarction operation, the level of miR328 in KD group was significantly lower than WT (Fig. 2B). To investigate whether the beneficial effects exist in vivo conditions, cardiac function was examined using echocardiography. As depicted in Fig. 2C, compared with WT+MI mice, ejection fraction (EF) and fractional shortening (FS) were significantly increased in $\mathrm{KD}+\mathrm{MI}$ hearts. Infarct size measurement by TTC staining showed that knockdown of miR-328 attenuated the deterioration of left ventricular infarct area (Fig. 2D). More importantly, collagen deposition in $\mathrm{KD}$ mice subjected to MI was significantly decreased and fibrosis declined to less than $20 \%$, compared to the WT+MI mice (Fig. 2E and $2 \mathrm{~F})$. These results demonstrated that cardiomyocytespecific overexpression of miR-328 promotes cardiac fibrosis, and the loss of miR328 ameliorated these fibrotic abnormalities.

TGF- $\beta$ signaling pathway mediates the pro-fibrotic effect of miR-328

The TGF- $\beta$ signaling pathway plays a vital role during the fibrotic pathological process [23]. In a previous study, we confirmed that TGF- $\beta$ RIII is also a direct target of miR-328 [24]. In addition, TGF- $\beta$ RIII, as a negative regulator for TGF- $\beta$ signaling, substantially neutralized TGF- $\beta 1$ and prevented TGF- $\beta$ RI/TGF- $\beta$ RII complex formation which, in turn, attenuated collagen production in cardiac fibroblasts [25]. In the present study, TGF- $\beta$ RIII mediated the pro-fibrotic effect of miR-328 through the inhibition of the TGF- $\beta$ pathway. As shown in Fig. 3A, the

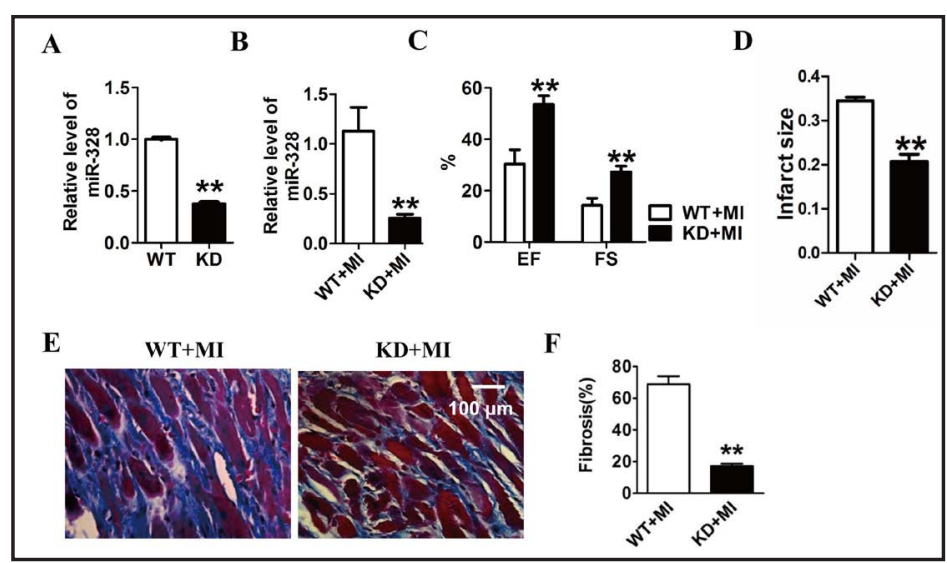

Fig. 2. Knockdown of endogenous miR-328 alleviates cardiac fibrosis in mice. (A-B) Decreased levels of miR-328 in KD mice compared with WT mice before and after MI. (C) Ejection fraction (\% EF) and fractional shortening (\% FS) were ameliorated in KD+MI mice, compared with WT+MI group. (D) Infarct size was reduced in KD mice following MI, compared with WT+MI mice. (E) Myocardial fibrosis in KD mice was attenuated during MI compared to WT mice. (F) Cross-sectional areas of fibrous tissue were analysed. $\mathrm{n}=5$ mice in each group. ${ }^{* *} \mathrm{p}<0.01$.

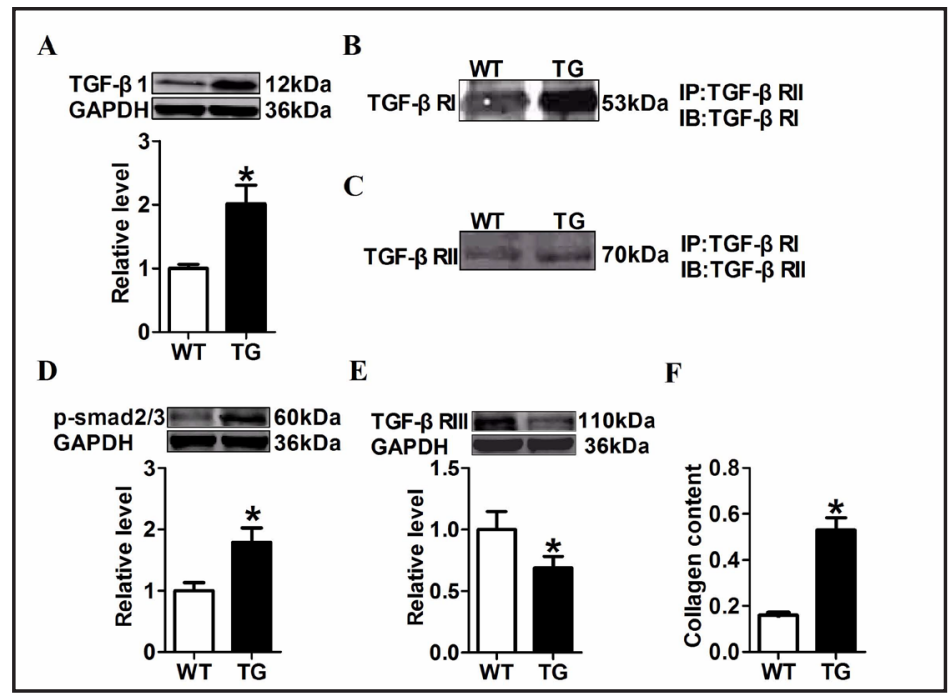

Fig. 3. Effects of miR-328 on the TGF- $\beta$ signaling pathway and on the collagen content. (A) TGF- $\beta 1$ was up-regulated in miR-328 TG mice. $\mathrm{n}=7$. (B-C) The interaction between TGF- $\beta$ RI and TGF- $\beta$ RII was enhanced in miR-328 TG mice, compared with WT. (D) The expression of p-smad2/3 was increased in miR-328 TG mice. $n=7$. (E) Down-regulation of TGF- $\beta$ RIII in miR-328 TG mice. $n=7$. (F) Increased collagen content in miR-328 TG mice. $n=5 .{ }^{*} \mathrm{p}<0.05$. 


\section{Cellular Physiology Cell Physiol Biochem 2018;46:1555-1565 \begin{tabular}{c|c} 
DOI: 10.1159/000489201 & O 2018 The Author(s). Published by S. Karger AG, Basel \\
www.karger.com/cpb
\end{tabular} \\ Zhao et al.: Cardiomyocytes-Derived MiR-328 Conduce to Cardiac Fibrosis}

protein level of TGF- $\beta 1$ was notably increased in TG mice. This increase was accompanied by the enhanced combination of TGF- $\beta$ RI/TGF- $\beta$ RII (Fig. 3B and 3C) and increased expression of p-smad2/3 (Fig. 3D), which act as signal transducers for the TGF- $\beta$ pathway. Additionally, western blot analyses showed that TGF- $\beta$ RIII was down-regulated in TG mice compared with WT mice (Fig. 3E). Additional studies showed that, compared with the collagen content in WT mice, the collagen content in TG mice was increased nearly 3-fold (Fig. 3F). These results suggested that the cardiomyocyte-specific overexpression of miR-328 increased the collagen content by inhibiting TGF- $\beta$ RIII and subsequently activated the TGF- $\beta$ signaling pathway.

Augmentation of miR-328 is dependent on translocation from CMs to CFs in a paracrine manner

To verify whether these effects of miR-328 on CFs reflect translocation from CMs, an in vitro co-culture assay system of CMs and CFs using transwell chambers was developed. CMs were seeded onto the upper transwell chambers and transfected with miR-328 mimics. After

Fig. 4. miR-328 is increased by vesicular transport in CFs. (A) CMs were transfected with miR-328 in transwell chambers. After $12 \mathrm{~h}$, the medium was changed, and the transfected CMs were shifted to co-culture with CFs for $48 \mathrm{~h}$. (B) miR-328 was elevated in transfected CMs, accompanied by an increase in the medium and in the co-cultured CFs. $n=5$. (C) The vesicles were detected in CMs using TEM. Apparent apoptosis, presented with karyopyknosis, membranolysis and mitochondrial degeneration, and increased vesicles were observed in CMs transfected with miR-328. $\mathrm{n}=3$. Arrowheads: extracellular vesicles; $\mathrm{N}$ : nucleus. (D) The viability of CMs were investigated with the method of CCK-8 assay. $\mathrm{n}=27$ samples from 3 different batches. (E) The level of miR-328 were elevated in the transfected co cultured CFs with or without Z-vad treatment. $\mathrm{n}=6$. (F) CFs were co-cultured with CMs transfected with Cy3-labelled miR-328 in transwell chambers. The luminescence of the co-cultured CFs was observed using immunofluorescence microscopy. Correspondingly, no luminescence was observed in CFs co-cultured with CMs without transfection. ${ }^{*} \mathrm{p}<0.05$, ${ }^{* *} \mathrm{p}<0.01$.

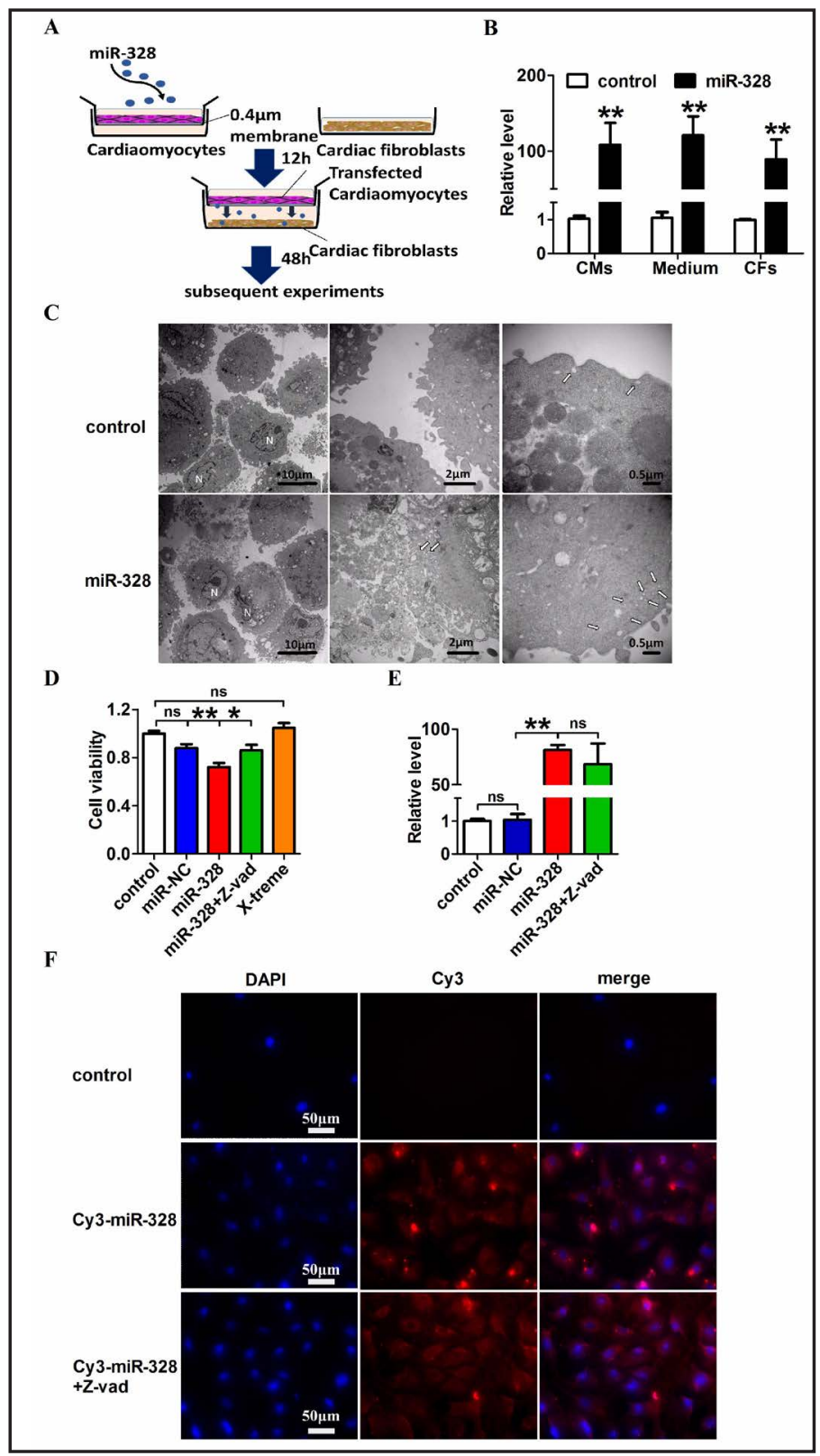




\section{Cellular Physiology Cell Physiol Biochem 2018;46:1555-1565 \begin{tabular}{ll|l} 
and Biochemistry Published online: April 24, 2018 & $\begin{array}{l}\text { (c) } 2018 \text { The Author(s). Published by S. Karger AG, Basel } \\
\text { www.karger.com/cpb }\end{array}$
\end{tabular}

$12 \mathrm{~h}$ of transfection, the medium was replaced, and transwells embedded with CMs were transferred to the culture flasks with CFs (Fig. 4A). Non-transfected CMs were cultured using the same protocol. After co-culture for $48 \mathrm{~h}$, the miR-328 content in the CMs, CFs and the medium were detected using real-time PCR analyses. As shown in Fig. 4B, the level of miR328 was markedly elevated approximately 100 -fold in transfected CMs compared with CMs without transfection (control). This increased expression was accompanied by an increase in the medium and in co-cultured CFs. Furthermore, electron microscopy images of the cellular ultrastructure showed an accumulation of vesicles (the diameter is about $40 \mathrm{~nm}-1000 \mathrm{~nm}$ ) secreted from cardiomyocytes transfected with miR-328. The vesicles observed in CMs were accompanied with apparent apoptotic changes (Fig. 4C). Considering that apoptosis assist in transport of miRNAs, Z-vad-FMK (Z-vad), an irreversible general caspase inhibitor, was added to inhibit the potential pro-apoptotic effects provoked by miR-328 in cardiomyocytes. As shown in Fig. 4D, Z-vad treatment significantly ameliorate CMs viability. More significantly, compared with the transfected miR-328 group, the addition of Z-vad did not reverse the level of miR-328 in the co-cultured CFs (Fig. 4E). To further validate the translocation of miR-328, CMs were transfected with Cy3-labelled miR-328 mimics. Red fluorescence was observed in CFs using immunofluorescence microscopy, whereas no luminescence was observed in the control group (Fig. 4F). The above results indicated that the increase of miR-328 is likely dependent on vesicular transport from CMs to $\mathrm{CFs}$ in a paracrine manner.

Transfer of miR-328 from CMs to CFs contributes to cardiac fibrosis via activating the TGF- $\beta$ signaling pathway

To further determine whether CM-derived miR-328 promotes fibrogenesis in CFs, the level of the fibrotic biomarkers Col $1 \alpha 1$ and Col3 $\alpha 1$ in co-cultured CFs were measured using real-time PCR. Expectedly, the up-regulation of the mRNA levels of Col $1 \alpha 1$ and Col3 $\alpha 1$ was

Fig. 5. The transfer of miR-328 from $\mathrm{CMs}$ to $\mathrm{CFs}$ contributes to cardiac fibrosis. (A-B) The RNA levels of Col $1 \alpha 1$ and Col3 $\alpha 1$ were increased in CFs when co-cultured with CMs transfected with miR328, determined using real-time PCR analysis. (C) The expression of Col1 $\alpha 1$ was up-regulated in CFs co-cultured with CMs transfected miR-328 but alleviated by AMO328 transfection in co-cultured CFs. (D) Representative images showing that the overexpression of miR-328 in CMs up-regulated the expression of $\alpha$-SMA and p-smad2/3 in co-cultured CFs using immunofluorescence analysis. (EG) Western blot analysis revealed that the expression of TGF- $\beta 1$ and p-smad $2 / 3$ was up-regulated, and the TGF- $\beta$ RIII level was correspondingly down-regulated in co-cultured CFs transfected with miR-328 into CMs. $\mathrm{n}=5 .{ }^{*} \mathrm{p}<0.05$.

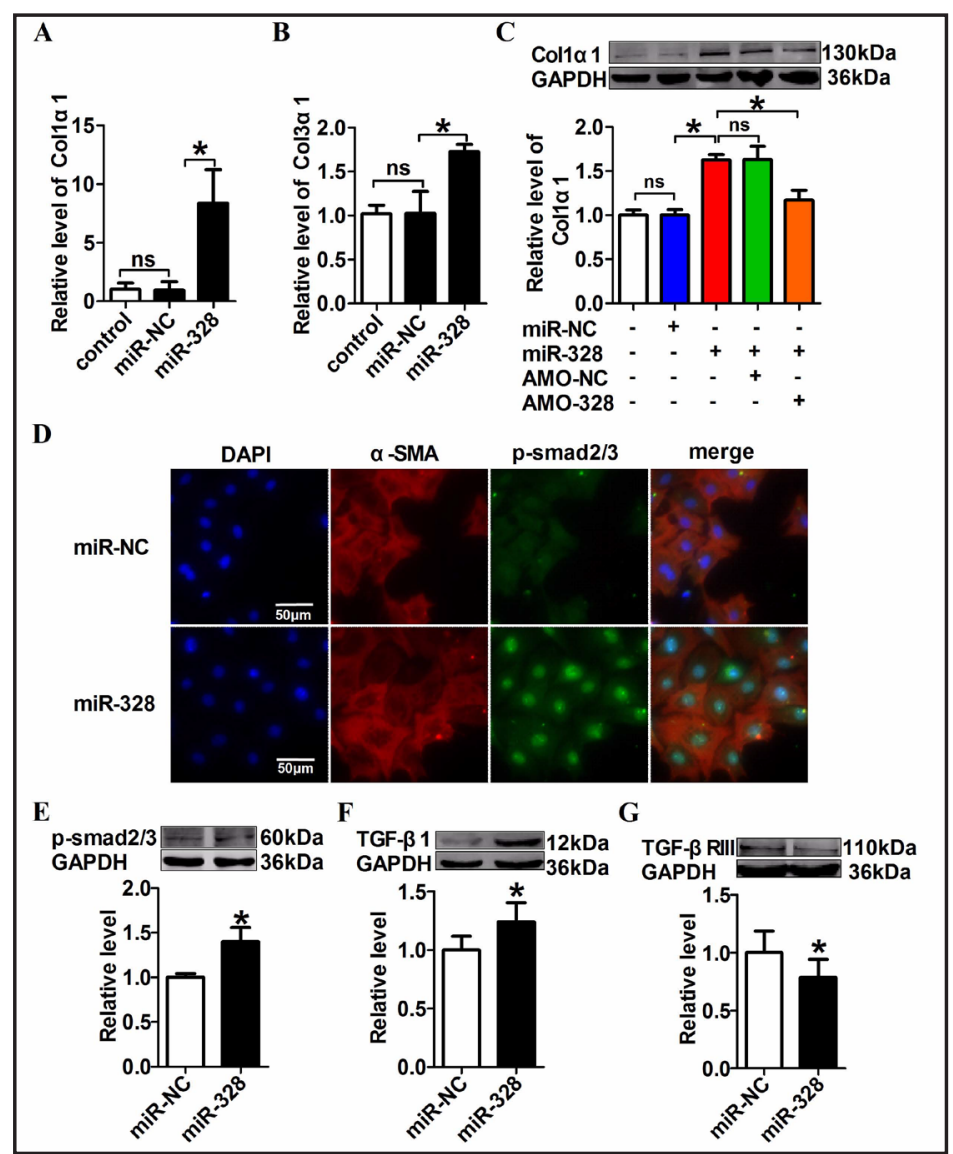




\section{Cellular Physiology Cell Physiol Biochem 2018;46:1555-1565 \\ \begin{tabular}{c|c} 
DOI: 10.1159/000489201 & O 2018 The Author(s). Published by S. Karger AG, Basel \\
www.karger.com/cpb
\end{tabular}}

Zhao et al.: Cardiomyocytes-Derived MiR-328 Conduce to Cardiac Fibrosis

remarkable following transfection of miR-328 (Fig. 5A and 5B). Notably, Col1 $\alpha 1$ protein expression was dramatically increased in CFs co-cultured with $\mathrm{CMs}$ transfected with miR-328, whereas co-application with AMO-328 to CFs exhibited the reverse effects on fibrogenesis in CFs, although AMO-NC had no effects (Fig. 5C). Immunofluorescence analysis showed that miR-328 overexpression in CMs up-regulated the expression of $\alpha$-smooth muscle actin ( $\alpha$-SMA) and p-smad2/3 in co-cultured CFs (Fig. 5D). Further investigation using western blotting analysis revealed that the expression of TGF- $\beta 1$ and p-smad $2 / 3$ was increased correspondingly with decreasing TGF- $\beta$ RIII level in CFs co-cultured with miR328 transfected CMs (Fig. 5E-5G). These results indicated that the miR-328 delivered from CMs to CFs activated the TGF- $\beta$ signaling pathway by inhibiting TGF- $\beta$ RIII and continuously initiating fibrogenic responses.

\section{Discussion}

Based on a previous study, the present study investigated whether cardiomyocytederived miR-328 promotes cardiac fibrosis by regulating adjacent fibroblasts in a paracrine manner. These findings showed that the cardiomyocyte-specific overexpression of miR-328 in TG mice caused robust fibrogenic responses, which promoted collagen production and induced fibrosis. We consistently observed that the normalization of miR-328 levels by antagomiR-328 and the knock down of endogenous miR-328 significantly abolished these fibrotic effects provoked by miR-328 and MI respectively. Additionally, the overexpression of miR-328 transport to CFs to stimulate the TGF- $\beta$ signaling pathway, including the upregulation of TGF- $\beta 1$ and p-smad $2 / 3$, and the down-regulation of TGF- $\beta$ RIII, ultimately lead to the enhancement of the collagen content.

The progression of cardiac fibrosis is governed through collaboration between cardiomyocytes and fibroblasts in the heart. Paracrine mediators, as an indirect communication strategy, could be excreted from one cell type to another, provoking corresponding effects $[26,27]$. miRNAs also can be on duty for a communication manner. miRNA transport way comprises with the aid of passive release of intracellular miRNAs from apoptotic cells and active release of intracellular miRNA in virtue of microvesicles, exosomes or apoptotic bodies. Ago2 protein-miRNA complex is also one of the modes of transport.

As previously reported, miR-328 governs atrial fibrillation via targeting CACNA1C and CACNB1 genes encoding L-type $\mathrm{Ca}^{2+}$ channel $\alpha 1 \mathrm{c}$ - and $\beta 1$-subunits [15] and equally promotes cardiac hypertrophy by enhancing intracellular $\mathrm{Ca}^{2+}$ level through translational inhibition of SERCA2a [14]. Calcium provokes the exocytosis of vesicles [28] suggesting that the transport of miR-328 is likely through vesicles. Relevant studies with respect to the role of miRNA transit in regulating cardiovascular pathogenesis have been published [29]. Bang $\mathrm{C}$ et al. reported that fibroblast-derived miR-21* (miR-21-3p, the passenger strands of miR21), as a critical paracrine mediator, contributes to the development of fibroblast-derived cardiomyocyte hypertrophy [13]. In the present study, evidence for miR-328 as a pro-fibrotic miRNA critically contributing to cardiac fibrosis via excretion of miR-328 from CMs to CFs emerged. Combined with previous reports, we proposed that the overexpression of miR-328 might stimulate CMs to secrete vesicles carrying miR-328 for transfer to CFs as paracrine manner. The vesicles in transfected CMs were observed by virtue of electron microscopy. Detected using real-time PCR and immunofluorescence, the transport of miR-328 from CMs to CFs did not be suppressed by Z-vad, which provided convincing evidence.

TGF- $\beta 1$ treatment potently stimulates collagen production to partially mediate cardiac fibrosis [30]. Active TGF- $\beta 1$ distinctly binds to the transmembrane TGF- $\beta R I I$, which is constitutively activated to form a heterotetrameric complex, which in turn recruits and interacts with TGF- $\beta$ RI. TGF- $\beta$ RI subsequently phosphorylates the downstream receptors Smad 2 and Smad 3 that further translocate to the nucleus and regulate the transcription of target genes [31]. Du et al. demonstrated that the overexpression of miR-328 repressed TGF- $\beta$ RIII and correspondingly promoted TGF- $\beta 1$ and TGF- $\beta$ RI/TGF- $\beta$ RII activation, which 


\section{Cellular Physiology Cell Physiol Biochem 2018;46:1555-1565 \begin{tabular}{l|l} 
DOI: 10.1159/000489201 & $\begin{array}{l}\text { O } 2018 \text { The Author(s). Published by S. Karger AG, Basel } \\
\text { www.karger.com/cpb }\end{array}$
\end{tabular}}

induced fibrous lesions [24]. In the present study, the transport of cardiomyocyte-derived miR-328 evoked fibrogenesis by suppressing TGF- $\beta$ RIII correspondingly activating TGF- $\beta$ signaling pathway in vivo and vitro.

Notably, although the present study showed that the overexpression of miR-328 derived from myocardial cells and translocated to myofibroblasts irritates the fibrotic response, the specific molecular mechanisms for the up-regulation of miR-328 expression in fibrotic pathological changes remain unknown. Moreover, cardiac fibrosis is the representative consequence of diversified cardiovascular diseases. Thus, distinguishing the role of AF and hypertrophy in fibrosis requires further detailed exploration.

\section{Acknowledgements}

This study was supported by the National Natural Science Foundation of China (31300943); Outstanding Youth Science Fund Project of Heilongjiang Province (JC201411); The China Postdoctoral Science Foundation (2016T90317), and the Wuliande Foundation of Harbin Medical University grant WLD-QN1707.

\section{Disclosure Statement}

The authors declared no conflict of interests.

\section{References}

1 Nguyen TP, Qu Z, Weiss JN: Cardiac fibrosis and arrhythmogenesis: the road to repair is paved with perils. J Mol Cell Cardiol 2014;70:83-91.

2 Miragoli M, Glukhov AV: Atrial Fibrillation and Fibrosis: Beyond the Cardiomyocyte Centric View: Biomed Res Int 2015;2015:798768.

-3 Cervio E, Barile L, Moccetti T, Vassalli G: Exosomes for Intramyocardial Intercellular Communication. Stem Cells Int 2015;2015:482171.

-4 Pellman J, Zhang J, Sheikh F: Myocyte-fibroblast communication in cardiac fibrosis and arrhythmias: Mechanisms and model systems. J Mol Cell Cardiol 2016;94:22-31.

5 Ongstad EL, Gourdie RG: Myocyte-fibroblast electrical coupling: the basis of a stable relationship? Cardiovasc Res 2012;93:215-217.

6 Yang B, Lin H, Xiao J, Lu Y, Luo X, Li B, Zhang Y, Xu C, Bai Y, Wang H, Chen G, Wang Z: The muscle-specific microRNA miR-1 regulates cardiac arrhythmogenic potential by targeting GJA1 and KCNJ2 Nat Med 2007;13:486-491.

7 Care A, Catalucci D, Felicetti F, Bonci D, Addario A, Gallo P, Bang ML, Segnalini P, Gu Y, Dalton ND, Elia L, Latronico MV, Hoydal M, Autore C, Russo MA, Dorn GW 2nd, Ellingsen O, Ruiz-Lozano P, Peterson KL, Croce CM, Peschle C, Condorelli G: MicroRNA-133 controls cardiac hypertrophy. Nat Med 2007;13:613-618.

8 Pan Z, Sun X, Shan H, Wang N, Wang J, Ren J, Feng S, Xie L, Lu C, Yuan Y, Zhang Y, Wang Y, Lu Y, Yang B: MicroRNA-101 inhibited postinfarct cardiac fibrosis and improved left ventricular compliance via the FBJ osteosarcoma oncogene/transforming growth factor- $\beta 1$ pathway. Circulation 2012;126:840-850.

-9 Xu S, Wang J, Ding N, Hu W, Zhang X, Wang B, Hua J, Wei W, Zhu Q: Exosome-mediated microRNA transfer plays a role in radiation-induced bystander effect. RNA Biol 2015;12:1355-1363.

-10 Climent M, Quintavalle M, Miragoli M, Chen J, Condorelli G, Elia L: TGF- $\beta$ Triggers miR-143/145 Transfer From Smooth Muscle Cells to Endothelial Cells, Thereby Modulating Vessel Stabilization. Circ Res 2015;116:1753-1764.

11 Aucher A, Rudnicka D, Davis DM: MicroRNAs transfer from human macrophages to hepato-carcinoma cells and inhibit proliferation. J Immunol 2013;191:6250-6260.

12 Alexander M, Hu R, Runtsch MC, Kagele DA, Mosbruger TL, Tolmachova T, Seabra MC, Round JL, Ward DM, O'Connell RM: Exosome-delivered microRNAs modulate the inflammatory response to endotoxin. Nat Commun 2015;6:7321. 


\section{Cellular Physiology Cell Physiol Biochem 2018;46:1555-1565 \begin{tabular}{l|l} 
DOI: 10.1159/000489201 & $\begin{array}{l}\text { O } 2018 \text { The Author(s). Published by S. Karger AG, Basel } \\
\text { www.karger.com/cpb }\end{array}$
\end{tabular}}

Zhao et al.: Cardiomyocytes-Derived MiR-328 Conduce to Cardiac Fibrosis

13 Bang C, Batkai S, Dangwal S, Gupta SK, Foinquinos A, Holzmann A, Just A, Remke J, Zimmer K, Zeug A, Ponimaskin E, Schmiedl A, Yin X, Mayr M, Halder R, Fischer A, Engelhardt S, Wei Y, Schober A, Fiedler J, Thum T: Cardiac fibroblast-derived microRNA passenger strand-enriched exosomes mediate cardiomyocyte hypertrophy. J Clin Invest 2014;124:2136-2146.

14 Li C, Li X, Gao X, Zhang R, Zhang Y, Liang H, Xu C, Du W, Zhang Y, Liu X, Ma N, Xu Z, Wang L, Chen X, Lu Y, Ju J, Yang B, Shan H: MicroRNA-328 as a regulator of cardiac hypertrophy. International Journal of Cardiology 2014;173:268-276.

15 Lu Y, Zhang Y, Wang N, Pan Z, Gao X, Zhang F, Zhang Y, Shan H, Luo X, Bai Y, Sun L, Song W, Xu C, Wang Z, Yang B: MicroRNA-328 contributes to adverse electrical remodeling in atrial fibrillation. Circulation 2010;122:2378-2387.

16 Yang B, Lin H, Xu C, Liu Y, Wang H, Han H, Wang Z: Choline produces cytoprotective effects against ischemic myocardial injuries: evidence for the role of cardiac M3 subtype muscarinic acetylcholine receptors. Cell Physiol Biochem 2005;16:163-174.

17 Liang H, Zhang C, Ban T, Liu Y, Mei L, Piao X, Zhao D, Lu Y, Chu W, Yang B: A novel reciprocal loop between microRNA-21 and TGF-ßRIII is involved in cardiac fibrosis. Int J Biochem Cell Biol 2012;44:2152-2160.

18 Liang H, Xu C, Pan Z, Zhang Y, Xu Z, Chen Y, Li T, Li X, Liu Y, Huangfu L, Lu Y, Zhang Z, Yang B, Gitau S, Lu Y, Shan H, Du Z: The antifibrotic effects and mechanisms of microRNA-26a action in idiopathic pulmonary fibrosis. Mol Ther 2014;22:1122-1133.

19 Gitau SC, Li X, Zhao D, Guo Z, Liang H, Qian M, Lv L, Li T, Xu B, Wang Z, Zhang Y, Xu C, Lu Y, Du Z, Shan H, Yang B: Acetyl salicylic acid attenuates cardiac hypertrophy through Wnt signaling. Front Med 2015;9:444456.

20 Chu W, Li C, Qu X, Zhao D, Wang X, Yu X, Cai F, Liang H, Zhang Y, Zhao X, Li B, Qiao G, Dong D, Lu Y, Du Z, Yang B: Arsenic-induced interstitial myocardial fibrosis reveals a new insight into drug-induced long QT syndrome. Cardiovasc Res 2012;96:90-98.

-21 He H, Liu X, Lv L, Liang H, Leng B, Zhao D, Zhang Y, Du Z, Chen X, Li S, Lu Y, Shan H: Calcineurin suppresses AMPK-dependent cytoprotective autophagy in cardiomyocytes under oxidative stress. Cell Death and Disease 2014;5:e997.

-22 Sun F, Duan W, Zhang Y, Zhang L, Qile M, Liu Z, Qiu F, Zhao D, Lu Y, Chu W: Simvastatin alleviates cardiac fibrosis induced by infarction via up-regulation of TGF- $\beta$ receptor III expression. Br J Pharmacol 2015;172:3779-3792.

23 Zhao X, Wang K, Liao Y, Zeng Q, Li Y, Hu F, Liu Y, Meng K, Qian C, Zhang Q, Guan H, Feng K, Zhou Y, Du Y, Chen Z: MicroRNA-101a inhibits cardiac fibrosis induced by hypoxia via targeting TGF- $\beta$ RI on cardiac fibroblasts. Cell Physiol Biochem 2015;35:213-226.

24 Du W, Liang H, Gao X, Li X, Zhang Y, Pan Z, Li C, Wang Y, Liu Y, Yuan W, Ma N, Chu W, Shan H, Lu Y: MicroRNA-328, a Potential Anti-Fibrotic Target in Cardiac Interstitial Fibrosis. Cell Physiol Biochem 2016;39:827-836.

25 Chu W, Li X, Li C, Wan L, Shi H, Song X, Liu X, Chen X, Zhang C, Shan H, Lu Y, Yang B: TGF- $\beta$ R3, a potential negative regulator of TGF- $\beta$ signaling, protects cardiac fibroblasts from hypoxia-induced apoptosis. J Cell Physiol 2011;226:2586-2594.

-26 Tsuruda T, Jougasaki M, Boerrigter G, Huntley BK, Chen HH, D’Assoro AB, Lee SC, Larsen AM, Cataliotti A, Burnett JC, Jr.: Cardiotrophin-1 stimulation of cardiac fibroblast growth: roles for glycoprotein 130/ leukemia inhibitory factor receptor and the endothelin type A receptor. Circ Res 2002;90:128-134.

-27 Barandon L, Dufourcq P, Costet P, Moreau C, Allieres C, Daret D, Dos Santos P, Daniel Lamaziere JM, Couffinhal T, Duplaa C: Involvement of FrzA/sFRP-1 and the Wnt/frizzled pathway in ischemic preconditioning. Circ Res 2005;96:1299-1306.

28 Subramanian J, Morozov A: ERK1/2 inhibit synaptic vesicle exocytosis through L-type calcium channels. J Neurosci 2011;31:4755-4764.

29 Das S, Halushka MK: Extracellular vesicle microRNA transfer in cardiovascular disease. Cardiovasc Pathol 2015;24:199-206.

-30 Ruiz-Ortega M, Rodriguez-Vita J, Sanchez-Lopez E, Carvajal G, Egido J: TGF- $\beta$ signaling in vascular fibrosis. Cardiovasc Res 2007;74:196-206.

31 Meng XM, Tang PM, Li J, Lan HY: TGF- $\beta$ /Smad signaling in renal fibrosis. Front Physiol 2015;6:82. 